\title{
ALFa - a software tool for optimal scheduling of demand oriented train services
}

\author{
S. Scholz \& T. Albrecht \\ Dresden University of Technology, Germany
}

\begin{abstract}
A scheduling strategy considering demand variation during the day and within the network is outlined. The optimal headway is calculated taking into account demand and supply simultaneously achieving equilibrium. The train service structure is complicated due to different headways for each hour of the day and various parts of the network. Short train headways shall be provided for sections with high demand whereas sections with lower demand (outskirts) request easily recallable departure times. That is why the use of Genetic Algorithms is proposed to transform such an operating scheme into an optimal train service. They allow to find a timetable with non-conflicting train paths, which is characterized by even headways for all sections of the network. The timetable can also be optimized for minimal fleet size or both goals simultaneously. In order to ease the application of such operating strategies, the proposed algorithms were implemented into a computer-aided planning system called ALFa which is described in this paper.
\end{abstract}

\section{Introduction}

Demand-driven train operation of fully automated underground railway lines can provide capacity strictly according to the fluctuation of demand. This helps avoid overcapacity during off-peak times and improves the revenue-cost-relation of the operator due to reduced operational efforts. Since this strategy has only been applied to single lines yet [1] the feasibility must be proven for a more extensive network of lines and their temporal and spatial demand distribution.

The basic approach of demand-driven operation is characterised by the control of the train headway $T_{S}$ in such a way that transport supply (capacity) is adapted to demand as much as possible. In the early morning and the late evening the longest train headway is chosen short enough so that the passengers do not need to 
know the timetable. Usually, a headway between six and ten minutes is used (cf. system VAL in Lille and Rennes or Skytrain in Vancouver). During the peak hours and during the day the train service is adapted to demand by varying the headway between one and six minutes (cf. upper left subfigure of Fig. 1).

Apart from this strategy to adapt to the temporal demand variation several operators of automated rapid transit systems also make use of a spatial adaptation. Short-turn trips serve only parts of the whole network and some sections of the network are commonly served by multiple lines. Furthermore, the vehicle size can be adopted in the course of the day or within the network (cf. Fig. 1). In practical operation even a combination of these four techniques is used. This paper focuses on the combination of temporal and spatial adaptation of the train headway.

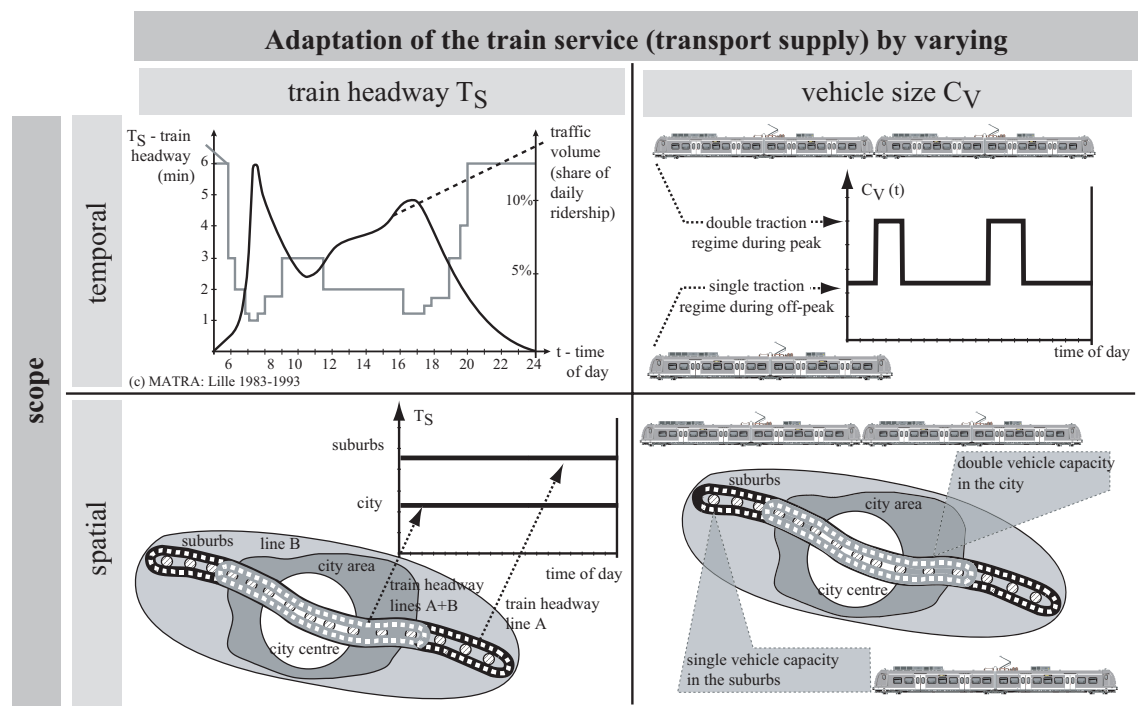

Figure 1: Adaptation of train service to demand - operational concepts.

Although a multitude of timetabling systems are described in the literature, none of them was able to solve the above problem of temporal and spatial adaptation of train headway on a network of lines in a satisfying, automatic manner. This paper presents a prototype of an integrated timetabling system, that has been developed at Dresden University of Technology and incorporates for the first time a supplydemand model and a timetabling algorithm in one tool. They will be described in section 2 and 3 of this paper. Section 4 and 5 present the developed software prototype and its first application. 


\section{Train service with supply-demand equilibrium}

The adjustment of supply to spatio-temporal variations in passenger demand helps improve basically the so called traffic efficiency [2]

$$
\eta=\frac{v l\left(T_{S}\right)}{b l\left(T_{S}, C_{V}, \gamma\right)} \cdot 100 \% \quad \hat{=} \frac{[v l]}{[b l]}=\frac{\text { pass. }-\mathrm{km} /(\text { year and track-km })}{\text { place-km } /(\text { year and track-km })} .
$$

The operational effort $b l$ is derived from the transport supply which is determined by the train headway and the vehicle size $C_{V}$ (regarding a maximum load factor $\gamma$ ). It can be adjusted to demand by choosing the optimal train headway $T_{S}$. Since line-haul rapid transit systems are considered, this train headway must be chosen with respect to the heaviest loaded section $x=\mu$ of a line. The traffic performance $v l$ (demand) is influenced by the quality of the train service, e.g. the train headway, too.

In order to achieve a high efficiency of the operation (cf. eq. 1) the transport supply and the transport demand must match optimally [2]. The maximum of the efficiency can be obtained by minimizing the deviation $\varepsilon_{V A G}$ between supply and demand at the heaviest loaded section of a line.

$$
\varepsilon_{V A G}\left(t, T_{S}, \mu\right)=\frac{C_{V} \cdot 60}{T_{S}} \cdot \gamma-V A\left(t, T_{S}, \mu\right) \rightarrow \mathrm{MIN} \geq 0 .
$$

Since demand and supply interact with each other, this supply-demand response must be considered in order to achieve consistent planning results. An aggregated transport demand model taking into account the response of demand to changes in supply was developed and described in detail in [3]. With the help of this model optimal train headways can be calculated that minimise overcapacity.

The dependency between demand and supply is illustrated in Fig. 2 for the suburban railway line of a medium sized conurbation [3]. It approx. corresponds to the city of Dresden, Germany and its suburbs. The interception point between supply and demand curve indicates a train headway with minimum overcapacity.

This transport model and the derived headway control strategy deliver the optimal train headway for each hour of the day and each branch of the network. This nominal train service structure with optimum supply-demand adaptation needs to be transfered into a feasible timetable with detailed departure times of the trains for each station.

\section{A Genetic Algorithm approach for automated timetabling}

\subsection{Problem description}

An optimal departure time within the given hour must be found for each of the obtained train rides. This is done in two stages:

1. Trains on lines with low operating frequencies are scheduled to fixed and easily recallable departure times (fixed headway over several hours). 


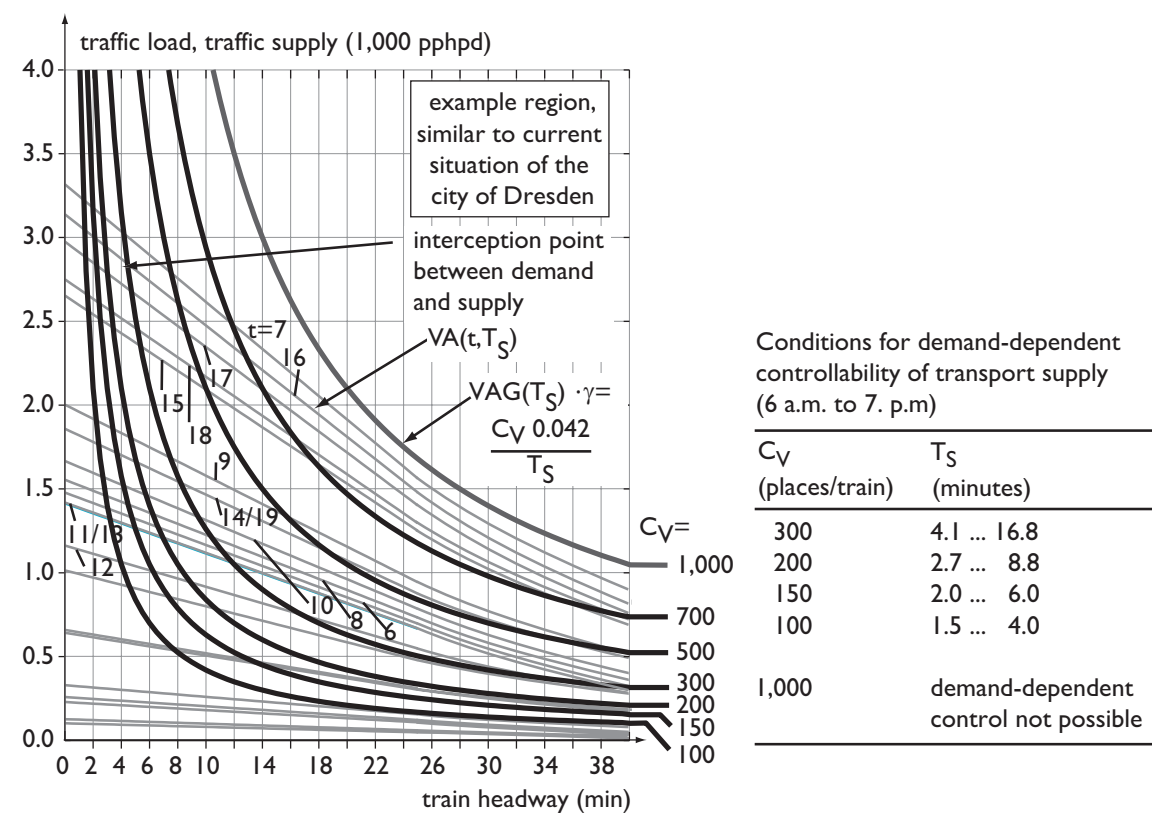

Figure 2: Conditions for demand-dependent controllability of transport supply [2].

2. Trains on all other lines, especially on those serving only small parts of the network, are scheduled in such a way, that train headway becomes as smooth as possible on every branch of the network and the necessary fleet size to realize this timetable becomes minimal.

The second task is very complicated, especially because of the long travel times on some of the lines. Those are the reason, that the global optimum of this problem can only be found by taking into consideration the whole operating day and not the single hours individually.

During the timetable design process, the most important constraint to be respected is minimal headway. It must be assured between suburban trains following each other as well as between suburban trains and trains of other operators running on the same infrastructure (freight or long distance passenger traffic).

\subsection{Computing optimal departure times}

Taking into account the nature of the cost function as well as the important number of variables, Genetic Algorithms (GA) are proposed to search for the optimal solution: This method has been described in detail in the literature $[4,5]$ and has become popular to solve complex railway optimization problems (e.g. [6, 7]). The fundamental way of solving technical problems by imitating the process of natural evolution is illustrated for the timetabling problem in Fig. 3. 


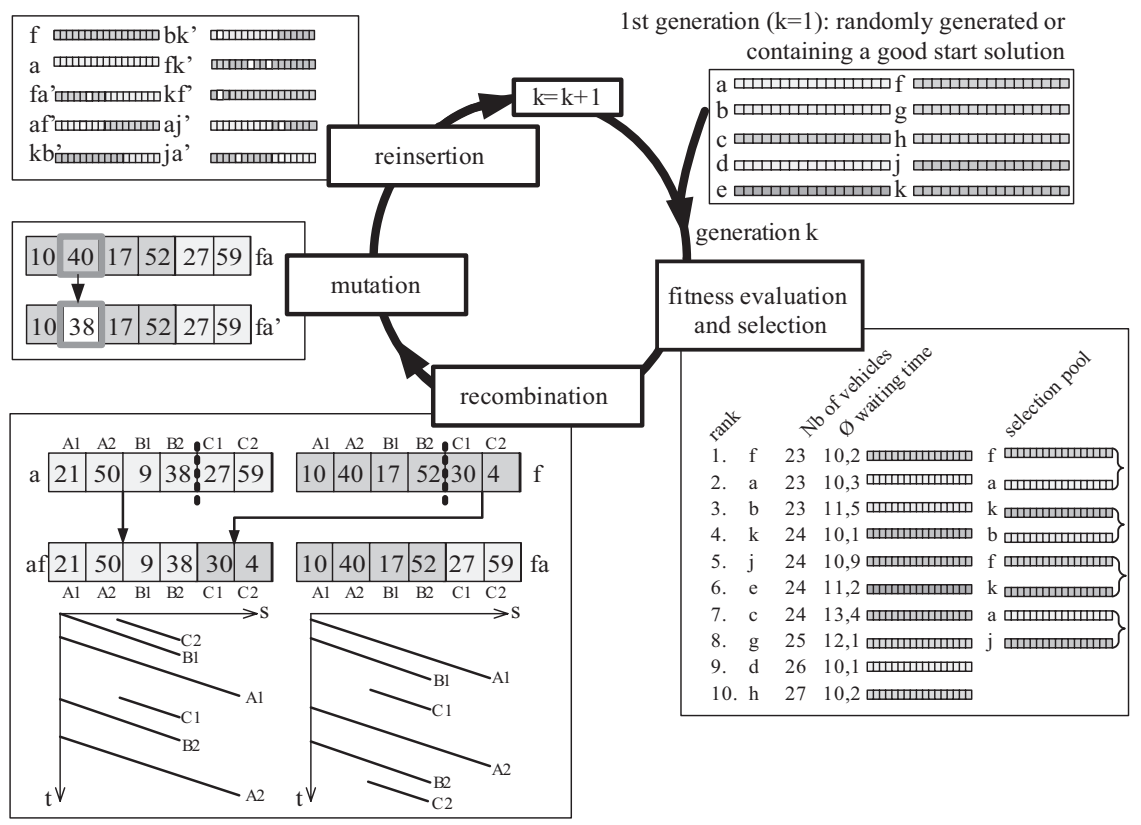

Figure 3: Using genetic algorithms for timetabling.

The most challenging point when using GA is to find a suitable coding of the problem to be solved. In Fig. 3, one way is described: the departure time (given in full minutes or part of minutes within the hour of the train's ride) is coded directly on a chromosome. When using this coding with the randomly acting GA operators, it is likely to happen that the timetable read from the chromosome contains departure times which do not respect the minimal headway. Those invalid timetables can either be penalized or transformed into feasible ones, which is proposed here.

Because of this problem of invalid solutions, an alternative coding could consist of taking the train headways as independent variables as well as the order of the lines. This coding reduces the number of conflicts drastically, but not to zero, because of the fixed departure times of trains of other operators that have to be respected. Both codings offer different advantages as was shown in [8].

The transformation process results in a timetable without conflicts. The cost function (passenger waiting time, fleet size) can now be calculated. Due to the nature of GA (which work on a representation of the solution and not directly on the parameters of the solution), other optimisation criteria ( $\mathrm{nb}$ of drivers, connections to other lines) can be easily incorporated without changing the algorithm or the coding. 


\section{Case study and test scenarios}

The outlined approach was implemented into a software prototype. The first application was the suburban railway network of Dresden, Germany. Here, the following operation scenarios have been tested (cf. Fig. 4):

- status quo scenario: situation of the year 2000 with network-wide headways of 20 to 30 minutes,

- M1-ITC scenario: traditional fixed interval timetable (peak: 7.5 min.; offpeak: 15 min.) and some short-turn trips during the peak period (track infrastructure: ITC - intermittent train control),

- M2-ITC scenario: advanced M1-ITC scenario with shortest train headways of 5 minutes and demand oriented train service in the city centre,

- M3-ETCS scenario: minimum train headways of 3.8 minutes, combination of temporal and spatial adaptation to demand in the urban area,

- AGT scenario: further enhanced M3 scenario assuming automated train operation with shortest headways of 2 minutes and network-wide combination of temporal and spatial adaptation to demand

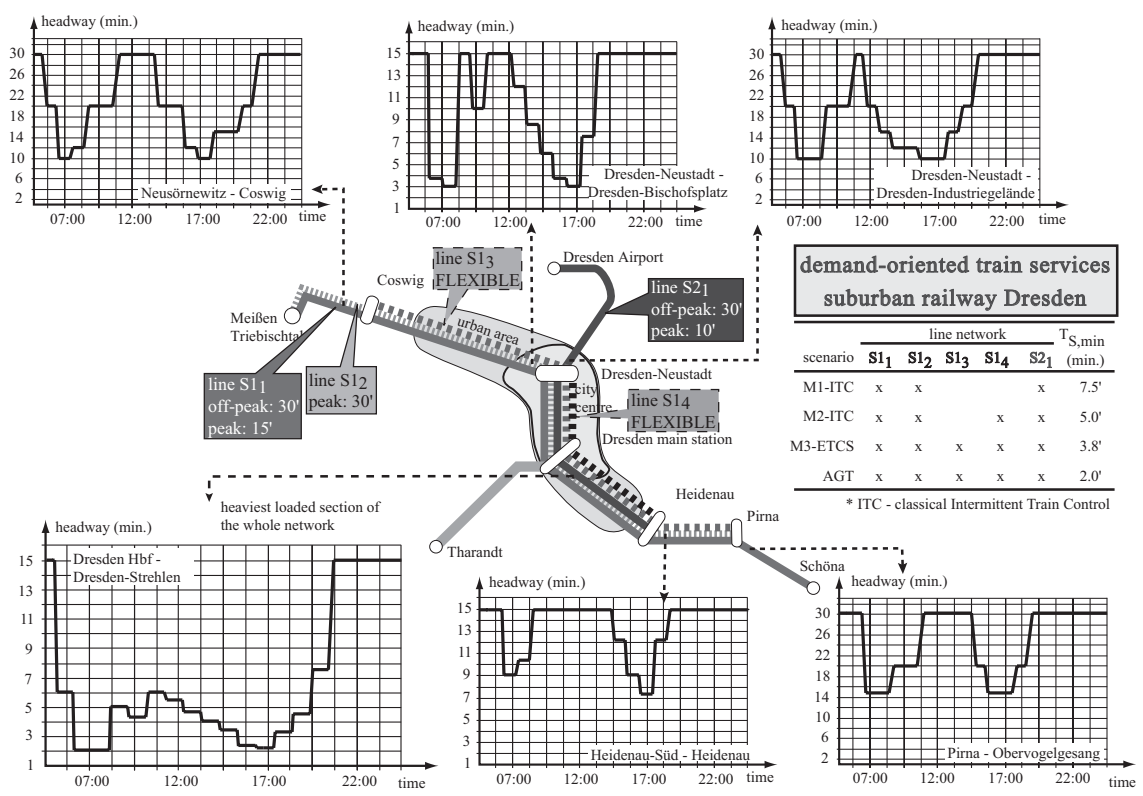

Figure 4: Train service structure: line network and headway for different scenarios. 


\section{Software prototype}

\subsection{Scope of application}

The developed software solution aims to solve three planning tasks:

1. ALFa-E: Event-based (re)-scheduling of existing timetables. The modification of existing timetables is especially important for hardly predictable changes in demand (weekend trips due to nice weather, shift from car to public transport due to frozen roads etc.). External short term demand forecasts can be used to adapt the nominal long term train service. Minor modifications include some extra trains or a reduction of the number of trains if possible. It can also be used in case of technical problems, e.g. blocked tracks due to switch failure.

2. ALFA-M: Medium term planning. The planning of demand-oriented train services several months in advance is considered as medium term planning. The nominal timetable for the next operating period can be adapted to average changes in demand in the course of the day and changes on weekdays and on weekends. The scenarios $M 1$ and $M 2$ belong to this category.

3. ALFa-S: Strategic planning. The third objective of the ALFa tool is dedicated to general planning issues with strategic goals and basic improvements due to major modifications of the train service and even the rolling stock. This fundamental modification must be considered a long term strategy requiring adequate fundamental studies on its expected benefits and operational feasibility. The scenarios $M 3$ and $A G T$ are examples of this sort of planning problems. Especially this issue requires to consider the supplydemand response of such basic improvements of the quality of train service. These fundamental modifications lead to highly complicated (time and space variant) train service structures which can hardly be transfered into feasible timetables with manual effort only.

\subsection{Functionality and graphical user interface}

The software prototype includes a transport demand module which enables the user to design the train services taking into account the demand reaction for that service' quality. Furthermore external demand data can be loaded through an interface (cf. Fig. 5). The train service is determined by the vehicle type of each line, the desired maximum load factor of the trains, the minimum and maximum admissible headways and the assumed supply-demand response. As a result the number of trains per hour for each section of the network is obtained.

Furthermore, all standard tasks for railway timetabling (cf. Fig. 6) such as: modeling of infrastructure, management of train running times and nominal station dwell times, support of various rolling stock, fixed timetables for certain lines (long-distance trains) and vehicle assignment are included. 


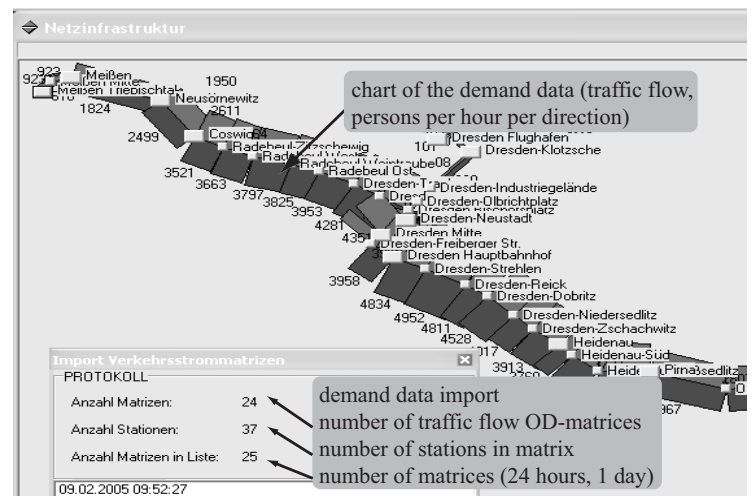

Figure 5: Network with demand distribution.

Apart from the implemented optimisation algorithms the software includes further functions that are primarily important for convenient user interaction. The calculated timetables can be displayed both as scalable time-distance-graph and spreadsheet (cf. Fig. 8) with export interface into standard EXCEL format.

The software manages all operational and infrastructural data which can be saved to different projects for easy modification and reuse.

The basic steps which need to be taken to calculate the timetable are as follows: definition of the track network with stations and interconnections, definition of a line network, definition of vehicle types, import of the transport demand (cf. Fig. 5) or explicit calculation of the required supply in order to meet demand, presetting of line parameters (planning constraints, cf. Fig. 7) and initiation of the automatic calculation of a timetable. An example timetable of the $A G T$ scenario is displayed in Fig. 8.

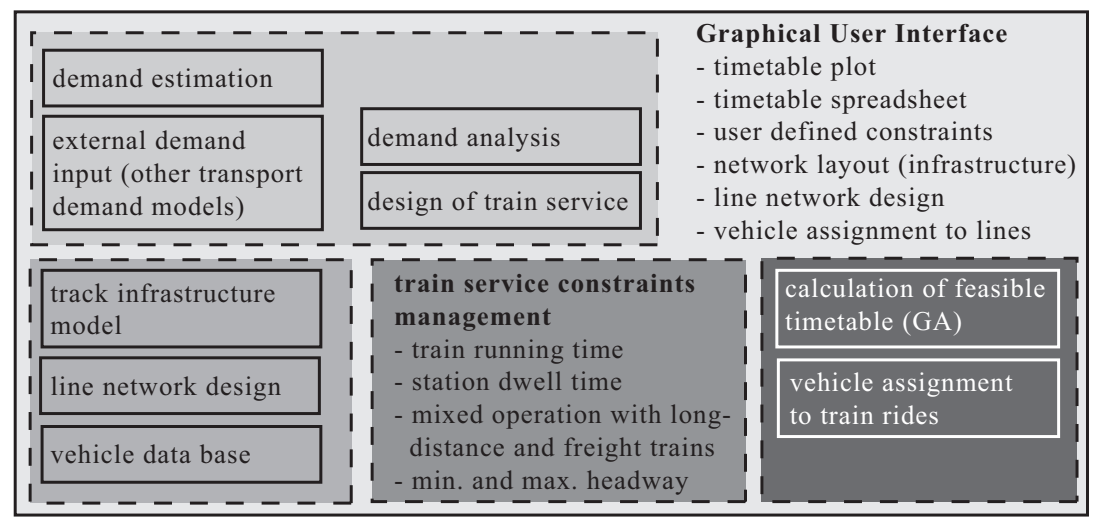

Figure 6: Modules of software prototype ALFa. 


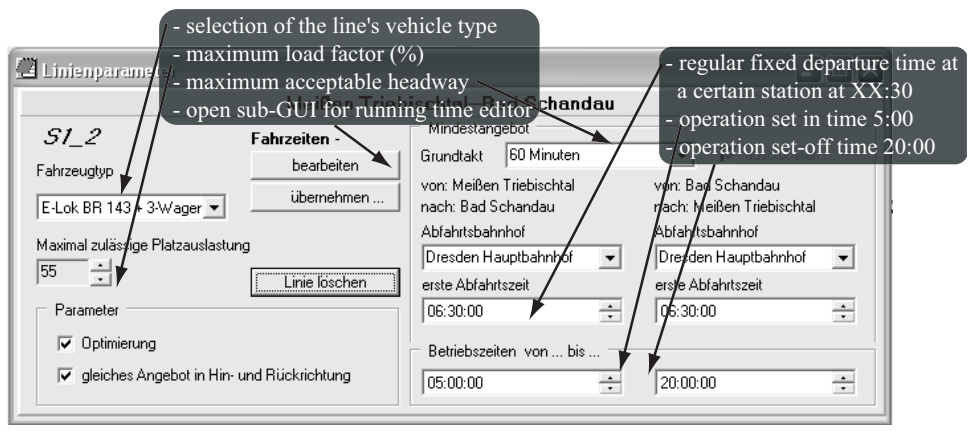

Figure 7: Basic line characteristics as calculation constraints.

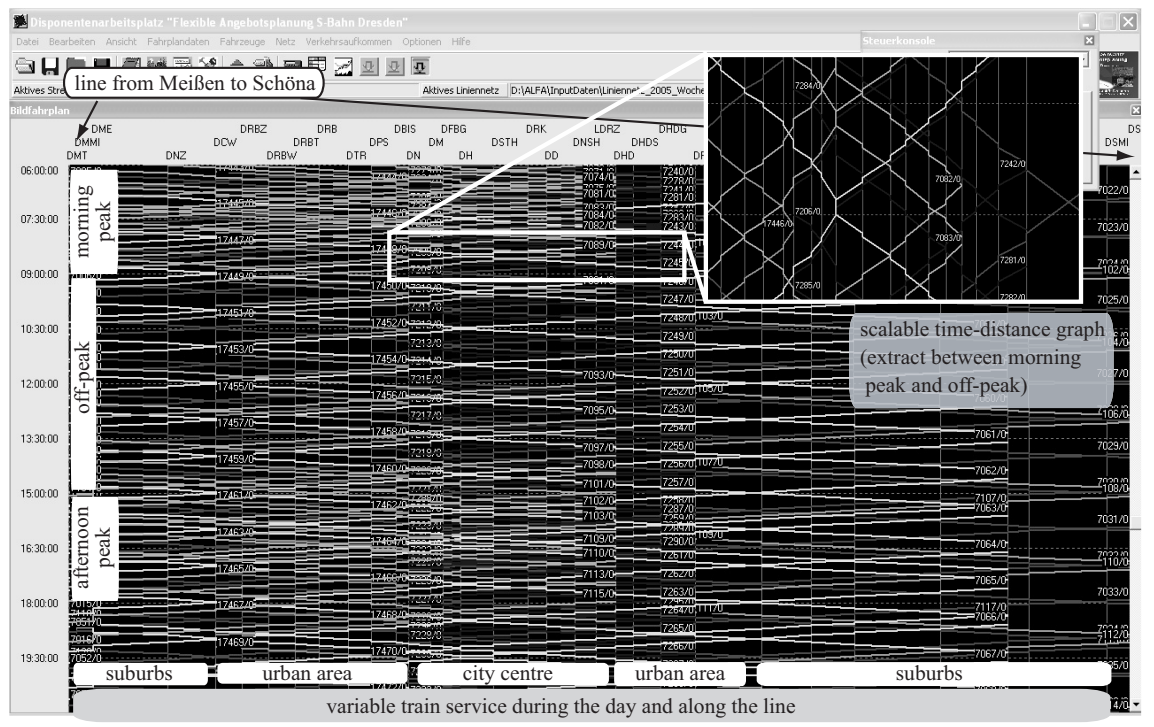

Figure 8: Time-distance train graph of the $A G T$ scenario.

\section{Conclusion}

The proposed compact transport demand model makes it possible to merge transport demand modeling and train service planning for the first time into a single train timetabling system. Planning of new and basically changed train services becomes more realistic and can be considered superior to straight-forward planning approaches with fixed demand. Genetic Algorithms are suitable to solve the difficult timetabling problem with the different operational constraints. The developed software prototype demonstrated its usefulness in several case studies for the suburban rapid rail system of a medium sized conurbation. 


\section{Acknowledgement}

ALFa was developed for DB Systems GmbH/ DB Regio AG within the research project "intermobil Region Dresden" funded by the German Federal Government, the ministry of Research and Education (BMBF). Project no.: 19 B 9907 A 8.

\section{References}

[1] N.N., Naissance d'un metro: The birth of a metro, 1998. Special issue: La vie du rail \& des transports.

[2] Scholz, S., Possibilities and limitations for a demand-dependent control of transport supply of commuter railways in medium-sized conurbations. Ph.D. thesis, University of Technology, Dresden, 2005. Dissertation.

[3] Scholz, S., Strobel, H. \& Oettich, S., Demand-driven automated urban rapid rail transit: a new approach to the assessment of the operational efficiency. $A P M{ }^{\prime} 03,9^{\text {th }}$ Int. Conf. on Automated People Movers, Singapore, 2003.

[4] Goldberg, D.E., Genetic Algorithms in Search, Optimisation and Machine Learning. Addison-Wesley: Reading, Massachussets, 1989.

[5] Whitley, D., A genetic algorithm tutorial. Statistics and Computing, 4, pp. 6585, 1994.

[6] Wegele, S. \& Schnieder, E., Automated dispatching of train operations using genetic algorithms. Computers in Railways IX, WIT Press, pp. 775-784, 2004.

[7] Kwan, R. \& Mistry, P., A co-evolutionary algorithm for train timetabling. 2003 Congress on Evolutionary Computation, IEEE, pp. 2142-2148, 2003.

[8] Albrecht, T., Nutzbarmachung Genetischer Algorithmen für den automatisierten Entwurf flexibler S-Bahn-Betriebsregime. at - Automatisierungstech$n i k, \mathbf{5 4 ( 3 )}$, pp. 1-8, 2006. 